\title{
Different Mechanisms of Ischemic Mitral Regurgitation in Patients With Inferior and Anterior Myocardial Infarction
}

\author{
Hui Zhang, MD*, Yutaka Otsuji, MD***, Takeshi Uemura, MD*, Bo Yu, MD*, \\ Masaaki Takeuchi, MD***, Shuichi Hamasaki, MD*, Masaaki Miyata, MD*, \\ Akira Kisanuki, MD*, Shinichi Minagoe, MD*, Robert A. Levine, MD** \\ and Chuwa Tei, MD* \\ *Department of Cardiovascular, Respiratory and Metabolic Medicine, Graduate School of \\ Medicine, Kagoshima University, Kagoshima, Japan \\ ${ }^{* *}$ Cardiac Ultrasound Laboratory, Massachusetts General Hospital, Boston, MA, USA \\ ${ }^{* * *}$ Second Department of Internal Medicine, University of Occupational and Environmental Health, \\ School of Medicine, Kitakyushu, Japan
}

\begin{abstract}
Background. Basic mechanism of ischemic mitral regurgitation (MR) is displacement of papillary muscles (PMs) due to left ventricular (LV) remodeling. Variability in LV remodeling can potentially cause heterogeneous PM displacements. The aim of this study is to compare the mitral valve complex geometry in patients with ischemic MR due to inferior and anterior myocardial infarction (MI).

Methods. In 33 patients with prior inferior MI, 61 with anterior MI, and 22 controls, LV volume, mitral annular area, PM tethering distance, and MR were quantified by echocardiography.

Results. Significant MR (MR fraction $>20 \%$ ) was observed in 12 of the 33 with inferior Ml and 7 of the 61 with anterior MI. In patients with MR due to inferior MI, tethering distance was significantly longer in medial compared to lateral PM (42.6 \pm 4.9 vs. $36.1 \pm 1.7 \mathrm{~mm}, \mathrm{p}<0.001)$, demonstrating asymmetric medial PM displacement. Patients with $\mathrm{MR}$ due to anterior $\mathrm{MI}$ had comparable increases in both PM tethering distances ( $41.0 \pm 2.4$ vs. $41.4 \pm 1.8 \mathrm{~mm}$, n.s.), demonstrating symmetric bilateral PM displacement.

Conclusions. Although patients with ischemic MR due to inferior MI have asymmetrically predominant medial PM displacement, those with ischemic MR due to anterior MI have symmetric bilateral PM displacements.
\end{abstract}

(J Echocardiogr 2008; 3: 74-83)

Key words: echocardiography, mitral regurgitation, left ventricular remodeling, ischemic heart disease

\section{Introduction}

Ischemic mitral regurgitation (MR) presents a challenging clinical problem associated with suboptimal surgical outcome and poor prognosis [1-7]. Augmented leaflet tethering by the outward displacement of papillary muscles (PMs) due to left ventricular

Received August 29, 2008; revision received October 10, 2008; accepted October 14, 2008

Address for correspondence: Yutaka Otsuji, MD

Second Department of Internal Medicine, University of Occupational and Environmental Health, School of Medicine,

1-1 Iseigaoka, Yahatanishi-ku, Kitakyushu, 807-8555, Japan.

Telephone: +81-93-691-7250

Fax: +81-93-691-6913

E-mail: otsujiy@med.uoeh-u.ac.jp

(C) 2008 Japanese Society of Echocardiography
(LV) remodeling is the basic mechanism of ischemic MR with apically displaced leaflets (Figure 1) [8-18]. Ischemic MR frequently develops in patients with inferior myocardial infarction (MI) and also in those with anterior MI [19-21]. Differences in the characteristics of $\mathrm{LV}$ remodeling in inferior and anterior MI suggests different mechanisms of ischemic MR in these patients. However, the differences in the mechanism are not fully investigated.

LV remodeling after inferior MI results in predominant displacement of the medial PM with only modest displacement of lateral PM or asymmetric medial PM displacement, which contributes to the development of ischemic MR (Figure 2, left panel) [12, 19]. In contrast, patients with anterior MI develop LV remodeling, which occurs predominantly in the anteroseptal and 


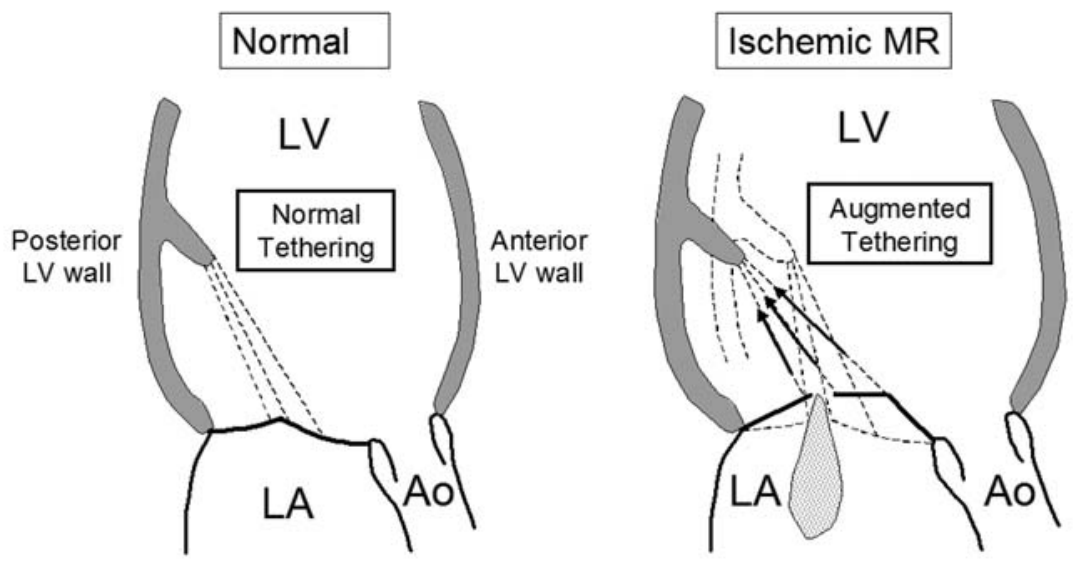

Fig. 1. A scheme illustrating the basic mechanism of ischemic mitral regurgitation (MR). Left ventricular (LV) remodeling causes outward displacement of papillary muscles (PM), which augments leaflet tethering and induce apical displacement of mitral leaflets and MR.

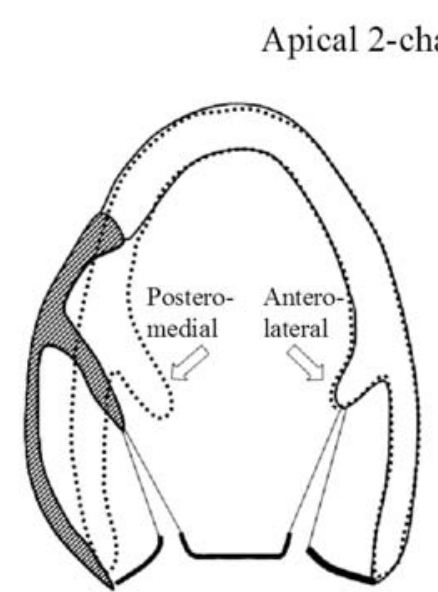

Inferior MI

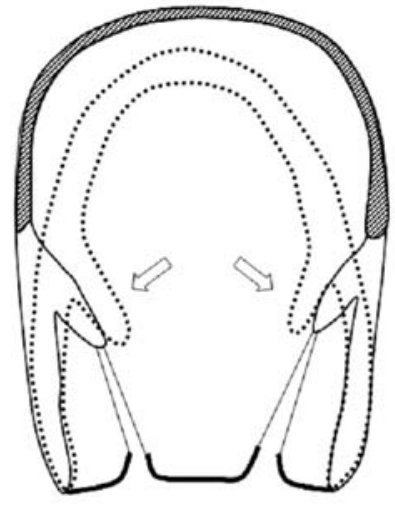

Anterior MI

Fig. 2. Potentially different mechanisms of ischemic MR due to inferior and anterior myocardial infarction (MI). LV remodeling after inferior $\mathrm{Ml}$ leads to predominant displacement of the medial PM with only modest displacement of lateral PM, resulting in asymmetric displacement of PMs with ischemic MR. In contrast, anterior $\mathrm{Ml}$ can potentially causes significant remodeling in anteroseptal and apical LV wall, resulting in symmetric displacement of both PMs to cause ischemic MR.

apical region and can potentially cause displacement of both PMs or symmetric PM displacement, resulting in a different mechanism of ischemic MR from that in inferior MI (Figure 2, right panel). Therefore, the purpose of this study is to compare the geometry of mitral valve complex in patients with significant ischemic MR due to inferior and anterior MI to test the hypothesis.

\section{Methods}

\section{Study population}

Subjects consisted of 22 normal control subjects, 33 consecutive patients with prior inferior MI, and 61 con- 
secutive patients with prior anteroseptal MI. Inclusion criteria was the presence of prior inferior or anteroseptal MI diagnosed on the basis of 1) a history of acute MI more than 1 month before with increased serum creatine kinase levels above twice the upper normal value and 2) segmental LV wall motion abnormality of the inferior or anteroseptal wall. Exclusion criteria were recent MI ( $<1$ month), multiple MIs, previous heart surgery, MR caused by organic mitral valvular lesions (including rheumatic changes, chordal or PM rupture, and infective endocarditis), and other associated cardiac diseases, such as aortic valve or congenital heart disease. Normal control subjects had normal echocardiograms without known cardiovascular disease. Written informed consent was obtained from all subjects and institutional committee of Kagoshima University approved the study protocol.

\section{Echocardiographic measurements}

All patients were referred for echocardiographic assessment between November 2002 and April 2003. Standard 2-dimensional and Doppler echocardiography examinations with color flow mapping were performed with the patients in the left lateral decubitus position using commercially available diagnostic systems (ATL HDI 3000, Bothell, Wash, USA; Toshiba SSH 380A, Tokyo, Japan; and Philips Medical Systems, Sonos 5500, Andover, Mass, USA). Special attention was paid to visualization of the lateral and medial PM tips in apical 4- and 2-chamber views. LV end-diastolic and end-systolic cavity areas were traced in those views, and LV volumes and ejection fractions (EFs) were obtained by using the modified biplane Simpson's method [22]. The LV wall was divided into $16 \mathrm{seg}$ ments, and the number of segments with wall motion abnormality was estimated to evaluate the spatial extent of ischemic LV dysfunction. Midsystolic mitral annular dimension was measured in the apical 4- and 2chamber views, and its area was calculated with an ellipsoid assumption (annular area $=\mathrm{d}_{1} \times \mathrm{d}_{2} \times \pi / 4$ ) $[23,24]$. The mitral annulus was identified as the leaflet hinge point. End-diastolic, end-systolic, and mid-systolic frames were determined as the initial, last, and middle frames with systolic mitral leaflet closure. The mitral leaflet tenting area between the leaflets and the line connecting the annular hinge points in the apical 4-chamber view was traced at mid-systole to estimate the apical displacement of the mitral leaflets [810]. The leaflet tethering distance between the PM tips and the contralateral anterior mitral annulus was also measured in the apical 4- and 2-chamber views by using the anterior mitral annulus as a reference point to estimate PM displacement (Figure 3) $[10,12,19]$. MR volume was calculated as the difference between mitral filling volume and aortic forward stroke volume obtained by the product of mitral or aortic annular area and the flow velocity time integral at the level using pulsed Doppler echocardiography [25]. Mitral filling volume was quantified as the difference between LV end-diastolic and end-systolic volumes in patients with suboptimal Doppler recordings and without aortic regurgitation [10]. MR fraction was then calculated as MR volume divided by mitral filling volume; MR fraction $\geq 20 \%$ was considered as significant [14].

\section{Reproducibility of measurements}

Two independent observers repeated measurements of PM tethering distance from 10 patients' echocardiographic recordings. The differences in their measurements were used to express interobserver variability. The same observer repeated the measurements to estimate intraobserver variability.

\section{Statistical analysis}

Results were expressed as means \pm SD. Variables were compared between groups by using the paired
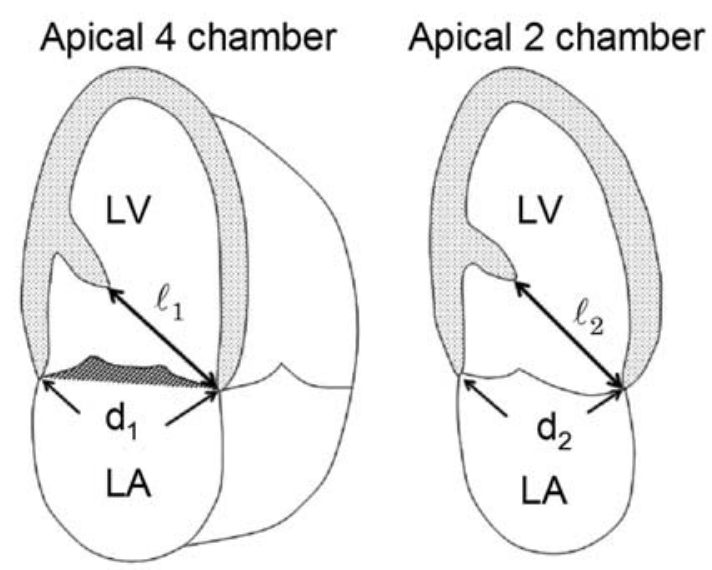

Fig. 3. Methods to measure 1) the mitral annular area from 2 diameters in apical views, 2) the mitral leaflet-tenting area between the mitral leaflet and a line connecting mitral annular hinge points in the mid-systolic apical 4-chamber view (dashed area shown in the left panel), and 3) papillary muscle (PM) leaflet-tethering lengths between lateral or medial PM tips and contralateral anterior mitral annulus $\left(\ell_{1}\right.$ and $\left.\ell_{2}\right)$. LV: Left ventricle, $\mathrm{LA}$ : left atrium, RV: right ventricle, RA: right atrium. 
and/or unpaired Student $t$ test. Incidences in 2 groups were compared by using the $\chi^{2}$ test. Determinants of the mitral leaflet tenting area and severity of MR in patients with inferior and anterior MI were explored by entering the end-diastolic and end-systolic LV volumes, $\mathrm{EF}$, mitral annular area, and PM-leaflet tethering distance into stepwise multiple regression analysis. A value of $p<0.05$ was considered statistically significant.

\section{Results}

Comparisons between entire patients with inferior and anterior MI (Table 1)

Compared to patients with inferior MI, patients with anterior MI had significantly greater LV dilatation and reduction in EF. Mitral annular area and lateral PM tethering length were significantly increased for both groups compared to controls but without significant difference between the 2 groups. In contrast, medial PM tethering length was significantly lengthened for both groups, and the elongation was significantly less in anterior compared to inferior MI. Consequently, the sum of tethering distances of both PMs was significantly greater in both groups than that of control group, but was again significantly less in patients with anterior MI. Increase in mitral leaflet tenting area as well as MR fraction were also significant in both groups, and these increases were again significantly less in anterior compared to inferior MI, which resulted in significantly lower incidence of significant MR in anterior compared to inferior MI. These 12 patients with significant ischemic MR due to inferior MI and 7 with MR due to anterior MI were enrolled to study subgroups for further comparison.

Table 1. Clinical and echocardiographic characteristics.

\begin{tabular}{|c|c|c|c|c|c|}
\hline & $\begin{array}{l}\text { Control } \\
(\mathrm{n}=22)\end{array}$ & $\begin{array}{l}\text { Inferior MI } \\
\quad(n=33)\end{array}$ & vs. & $\begin{array}{l}\text { Anterior MI } \\
\quad(n=61)\end{array}$ & $p$ Value \\
\hline Age $(y)$ & $65 \pm 12$ & $66 \pm 9$ & & $66 \pm 11$ & n.s. \\
\hline Gender $(M / F)$ & $18 / 4$ & $28 / 5$ & & $54 / 7$ & n.s. \\
\hline Heart failure & $0 / 22$ & $4 / 33$ & & $6 / 61$ & n.s. \\
\hline $\begin{array}{l}\text { Time lapse } \\
\text { after acute MI (mo) }\end{array}$ & $0 \pm 0$ & $29 \pm 35$ & & $27 \pm 32$ & n.s. \\
\hline LVEDV $(m l)$ & $91 \pm 11$ & $106 \pm 19$ & & $125 \pm 45$ & $<0.01$ \\
\hline LVESV $(m l)$ & $38 \pm 13$ & $52 \pm 30$ & & $71 \pm 34$ & $<0.01$ \\
\hline LVEF (\%) & $63 \pm 6$ & $49 \pm 8$ & & $44 \pm 7$ & $<0.01$ \\
\hline $\begin{array}{l}\text { Number of LV segments } \\
\text { with asynergy }\end{array}$ & $0 \pm 0$ & $1.8 \pm 0.7$ & & $5.8 \pm 2.2$ & $<0.001$ \\
\hline $\begin{array}{l}\text { Mitral Annular Area } \\
\left(\mathrm{cm}^{2}\right)\end{array}$ & $7.8 \pm 0.8$ & $9.2 \pm 1.3$ & & $8.9 \pm 1.4$ & n.s. \\
\hline \multicolumn{6}{|l|}{ PM Tethering Distance } \\
\hline$\ell_{1}($ lateral PM) $(\mathrm{mm})$ & $33.5 \pm 2.2$ & $35.5 \pm 1.9$ & & $35.1 \pm 3.3$ & n.s. \\
\hline$\ell_{2}($ medial PM) $(\mathrm{mm})$ & $33.4 \pm 2.1$ & $38.8 \pm 4.9$ & & $34.9 \pm 3.3$ & $<0.001$ \\
\hline$\ell_{1}+\ell_{2}(\mathrm{~mm})$ & $66.9 \pm 3.7$ & $74.1 \pm 5.6$ & & $70.0 \pm 6.4$ & $<0.001$ \\
\hline $\begin{array}{l}\text { Mitral Tenting Area } \\
\left(\mathrm{cm}^{2}\right)\end{array}$ & $0.11 \pm 0.08$ & $0.76 \pm 0.52$ & & $0.58 \pm 0.35$ & $<0.05$ \\
\hline MR fraction (\%) & $2 \pm 5$ & $17 \pm 13$ & & $7 \pm 9$ & $<0.001$ \\
\hline $\begin{array}{l}\text { Incidence of } \\
\text { MR Fraction } \geq 20 \%\end{array}$ & 0 / $22(0 \%)$ & 12 / $33(36 \%)$ & & 7 / 61 (11\%) & $<0.01$ \\
\hline
\end{tabular}

MI: myocardial infarction, LVEDV: left ventricular end-diastolic volume, LVESV: left ventricular end-systolic volume, LVEF: left ventricular ejection fraction, $\ell_{1}$ : lateral PM tethering distance, $\ell_{2}$ : medial PM tethering distance, MR: mitral regurgitation. 
Comparisons between patients with significant MR due to inferior and anterior MI (Table 2)

Comparisons between patients with significant MR due to inferior and anterior MI revealed that increase in LV end-diastolic and end-systolic volume and reduction in LVEF were significantly greater in ischemic MR due to anterior MI compared to inferior MI. Mitral annular dilatation was comparable. Elongation of lateral PM tethering distance was greater in ischemic MR due to anterior MI whereas the increase in medial and sum of PM tethering distances were comparable. These resulted in predominant or asymmetric increase in medial PM tethering distance in MR due to inferior MI and symmetric increase in both PM tethering dis- tances in MR due to anterior MI (Figure 4). Although mitral leaflet tenting area was comparable between the 2 groups, MR fraction was significantly less in significant MR due to anterior MI compared to the MR due to inferior MI, suggesting that asymmetric PM displacement may cause greater MR when the sum of PM tethering was comparable.

Figure 5 shows two representative patients: one with ischemic MR due to inferior MI associated with asymmetric and predominant increase in medial PM tethering distance and another with ischemic MR due to anterior MI associated with symmetric increase in both PM tethering distances.

Table 2. Comparisons between patients with ischemic MR due to inferior and anterior MI.

\begin{tabular}{|c|c|c|c|c|}
\hline MR & $\begin{array}{l}\text { due to Inferior MI } \\
\qquad(\mathrm{n}=12)\end{array}$ & vs. & $\begin{array}{l}\text { MR due to Anterior MI } \\
\qquad(\mathrm{n}=7)\end{array}$ & $p$ Value \\
\hline LVEDV $(m l)$ & $126 \pm 11$ & & $197 \pm 63$ & $<0.05$ \\
\hline LVESV (ml) & $76 \pm 25$ & & $128 \pm 43$ & $<0.001$ \\
\hline LVEF (\%) & $46 \pm 6$ & & $36 \pm 4$ & $<0.01$ \\
\hline Mitral Annular Area $\left(\mathrm{cm}^{2}\right)$ & $10.5 \pm 1.4$ & & $9.7 \pm 1.1$ & n.s. \\
\hline \multicolumn{5}{|l|}{ PM Tethering Distance } \\
\hline$\ell_{1}(\mathrm{~mm})$ & $36.1 \pm 1.7$ & & $41.4 \pm 1.8$ & $<0.05$ \\
\hline$\ell_{2}(m m)$ & $42.6 \pm 4.9$ & & $41.0 \pm 2.4$ & n.s. \\
\hline$\ell_{1}+\ell_{2}(\mathrm{~mm})$ & $78.7 \pm 6.0$ & & $82.4 \pm 3.8$ & n.s. \\
\hline Mitral Tenting Area $\left(\mathrm{cm}^{2}\right)$ & $1.3 \pm 0.4$ & & $1.2 \pm 0.2$ & n.s. \\
\hline MR fraction (\%) & $32 \pm 9$ & & $27 \pm 5$ & $<0.001$ \\
\hline
\end{tabular}

Abbreviations: as in Table 1.

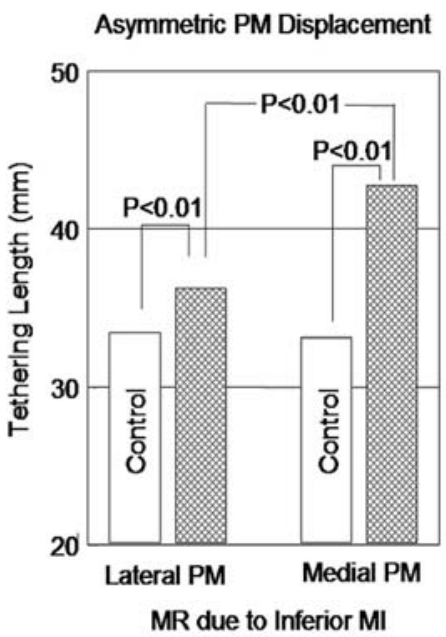

Symmetric PM Displacement

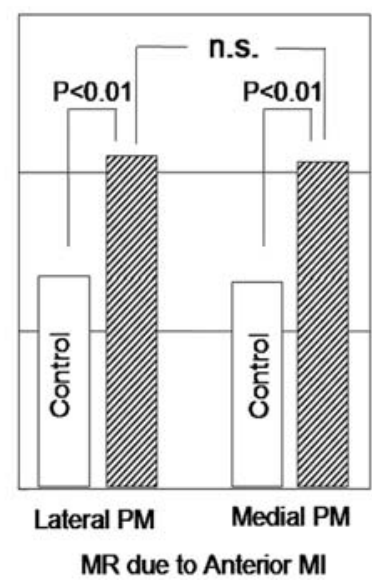

Fig. 4. Bar graphs showing predominant and asymmetric increase in tethering distance of medial papillary muscle (PM) in ischemic MR with inferior MI (left panel) and symmetric increases in tethering distance of both PMs in ischemic MR with anterior MI (right panel). 

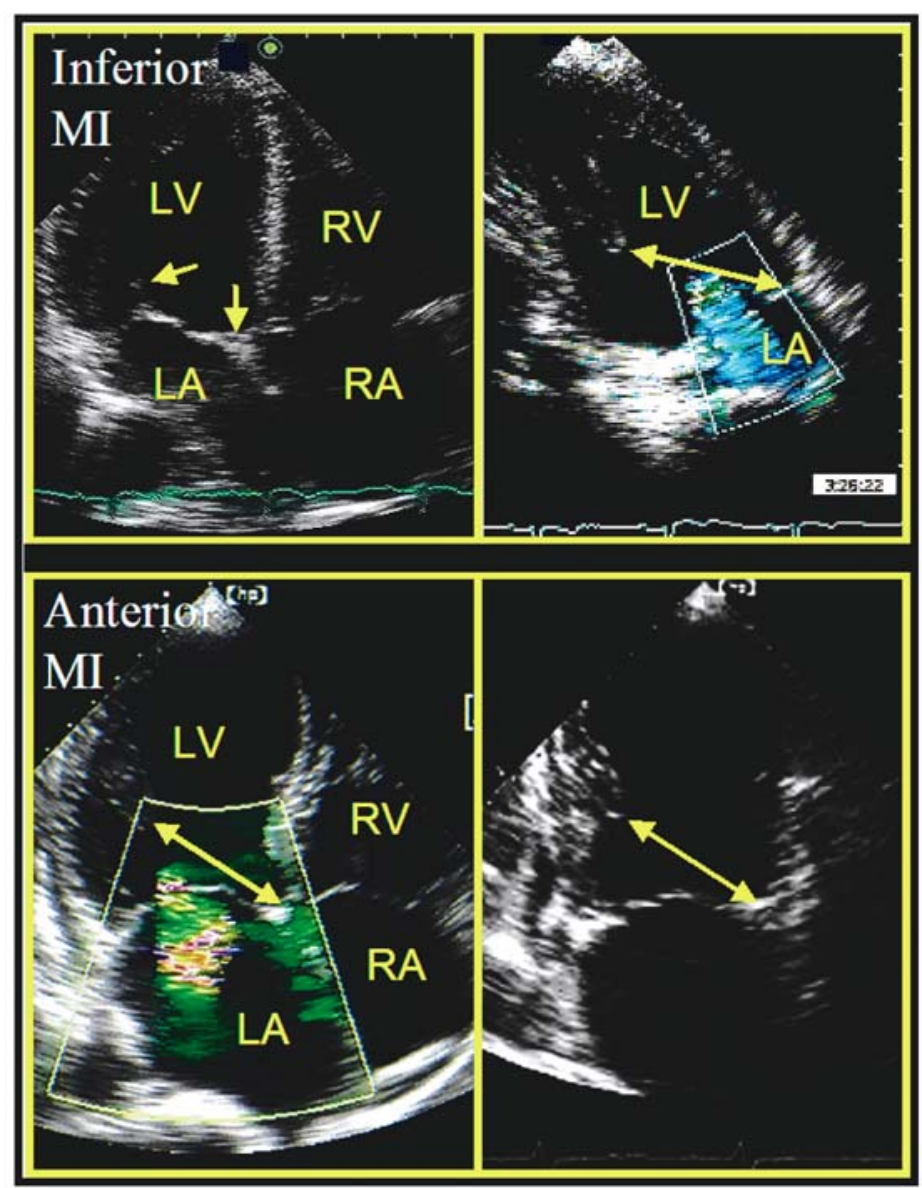

Fig. 5. The patient with ischemic MR due to inferior MI (upper panels) showed an asymmetric displacement of PMs with predominantly longer tethering distance of medial (upper-right panel) compared to lateral PM. In contrast, symmetric displacement of both PMs was demonstrated in another patient with ischemic MR due to anterior MI with comparably elongated tethering distance of both PMs (lower panels).

\section{Determinants of mitral leaflet tenting area and MR fraction}

Multiple geometric indices showed significant relations with mitral leaflet tenting area in both patients with inferior as well as anterior MI by univariate analysis (Table 3). Multivariate analysis identified increase in medial PM tethering distance along with increase in LVEDV as the independent determinants of the leaflet tenting area in patients with inferior MI. In contrast, the increase in the sum of PM tethering distances was selected as the only independent determinant of the tenting area in patients with anterior MI. These suggest that asymmetric medial PM displacement is the major determinant of mitral leaflet tenting area in patients with inferior MI, while symmetric displacement of both PMs is the determinant in anterior MI.

Similarly, multiple geometric indices showed significant relations with MR fraction in both patients with inferior as well as anterior MI by univariate analysis (Table 4). Multivariate analysis identified increase in medial PM tethering distance along with increase in LVEDV as the independent determinant of MR fraction in patients with inferior MI, while the sum of both PM tethering distances along with increase in LVESV were selected as independent determinants in patients with anterior MI. These results again suggest that asymmetric medial PM displacement is the major determinant of MR fraction in patients with inferior MI, while 
Table 3. Determinants of mitral leaflet tenting area.

\begin{tabular}{|c|c|c|c|c|}
\hline \multirow[t]{2}{*}{ Univariate Analysis } & \multicolumn{2}{|c|}{ Inferior MI $(\mathrm{n}=33)$} & \multicolumn{2}{|c|}{ Anterior MI $(\mathrm{n}=61)$} \\
\hline & $r^{2}$ & $p$ value & $r^{2}$ & $p$ value \\
\hline LV EDV & 0.48 & $<0.0001$ & 0.39 & $<0.0001$ \\
\hline LV ESV & 0.25 & $<0.005$ & 0.38 & $<0.0001$ \\
\hline LV EF & 0.04 & n.s. & 0.17 & $<0.001$ \\
\hline $\mathrm{LAD}$ & 0.11 & n.s. & 0.06 & n.s. \\
\hline Mitral Annular Area & 0.25 & $<0.005$ & 0.07 & $<0.05$ \\
\hline$\ell_{1}$ & 0.12 & $<0.05$ & 0.58 & $<0.0001$ \\
\hline$\ell_{2}$ & 0.65 & $<0.0001$ & 0.66 & $<0.0001$ \\
\hline$\ell_{1}+\ell_{2}$ & 0.59 & $<0.0001$ & 0.67 & $<0.0001$ \\
\hline Multivariate Analysis & Coefficient & $p$ value & Coefficient & $p$ value \\
\hline LV EDV & 0.01 & $<0.0001$ & $(-)$ & n.s. \\
\hline LV ESV & $(-)$ & n.s. & $(-)$ & n.s. \\
\hline LV EF & $(-)$ & n.s. & $(-)$ & n.s. \\
\hline $\mathrm{LAD}$ & $(-)$ & n.s. & $(-)$ & n.s. \\
\hline Mitral Annular Area & $(-)$ & n.s. & $(-)$ & n.s. \\
\hline$\ell_{1}$ & $(-)$ & n.s. & $(-)$ & n.s. \\
\hline$\ell_{2}$ & 0.07 & $<0.0001$ & $(-)$ & n.s. \\
\hline$\ell_{1}+\ell_{2}$ & $(-)$ & n.s. & 0.04 & $<0.0001$ \\
\hline
\end{tabular}

Abbreviations: as in Table 1.

Table 4. Determinants of MR fraction.

\begin{tabular}{|c|c|c|c|c|}
\hline \multirow[t]{2}{*}{ Univariate Analysis } & \multicolumn{2}{|c|}{ Inferior MI ( $\mathrm{n}=33)$} & \multicolumn{2}{|c|}{ Anterior MI $(\mathrm{n}=61)$} \\
\hline & $r^{2}$ & $p$ value & $r^{2}$ & $p$ value \\
\hline LV EDV & 0.59 & $<0.0001$ & 0.38 & $<0.0001$ \\
\hline LV ESV & 0.39 & $<0.0001$ & 0.42 & $<0.0001$ \\
\hline LV EF & 0.11 & n.s. & 0.25 & $<0.001$ \\
\hline $\mathrm{LAD}$ & 0.24 & $<0.005$ & 0.09 & $<0.05$ \\
\hline Mitral Annular Area & 0.39 & $<0.0005$ & 0.44 & $<0.0004$ \\
\hline$\ell_{1}$ & 0.13 & $<0.05$ & 0.65 & $<0.0001$ \\
\hline$\ell_{2}$ & 0.64 & $<0.0001$ & 0.62 & $<0.0001$ \\
\hline$\ell_{1}+\ell_{2}$ & 0.59 & $<0.0001$ & 0.67 & $<0.0001$ \\
\hline Multivariate Analysis & Coefficient & $p$ value & Coefficient & $p$ value \\
\hline LV EDV & 0.33 & $<0.0001$ & $(-)$ & n.s. \\
\hline LV ESV & $(-)$ & n.s. & 0.001 & $<0.0001$ \\
\hline LV EF & $(-)$ & n.s. & $(-)$ & n.s. \\
\hline $\mathrm{LAD}$ & $(-)$ & n.s. & $(-)$ & n.s. \\
\hline Mitral Annular Area & $(-)$ & n.s. & $(-)$ & n.s. \\
\hline$\ell_{1}$ & $(-)$ & n.s. & $(-)$ & n.s. \\
\hline$\ell_{2}$ & 1.60 & $<0.0001$ & $(-)$ & n.s. \\
\hline$\ell_{1}+\ell_{2}$ & $(-)$ & n.s. & 0.011 & $<0.0001$ \\
\hline
\end{tabular}

Abbreviations: as in Table 1.

PM displacement is the determinant in those with anterior MI.

\section{Reproducibility of measurements}

Inter- and intra-observer variabilities for the measurements of PM leaflet-tethering distance were $1.5 \pm$ 0.8 and $0.9 \pm 0.5 \mathrm{~mm}$ or $4.1 \pm 2.2 \%$ and $2.3 \pm 1.5 \%$ of the mean value, respectively.

\section{Discussion}

Different mechanisms of ischemic MR due to inferior and anterior MI

The basic mechanism of ischemic MR is leaflet teth- 
ering due to outward displacement of PMs with LV remodeling (Figure 1) [8-21]. Both inferior as well as anterior MI cause ischemic MR [19-21]. Because the characteristics of $\mathrm{LV}$ remodeling are different between patients with inferior and anterior MI, it is expected that ischemic MR due to inferior and anterior MI may have different mechanisms. Asymmetric and predominant displacement of medial PM has been shown to cause ischemic MR in inferior MI (Figure 2) [12, 1921]. However, the mechanism of ischemic MR due to anterior MI has not been fully investigated. Broad involvement of LV remodeling, including the apex and anteroseptal wall, in anterior MI suggests that symmetric displacement of both PMs can be the mechanism of its ischemic MR (Figure 2). The present study demonstrated asymmetrically elongated medial PM tethering distance in patients with ischemic MR due to inferior MI and symmetrically elongated medial and lateral PM tethering distances in patients with the MR due to anterior MI. Therefore, the present study demonstrated that leaflet tethering is the basic mechanism of ischemic MR both in inferior and anterior MI. However, the nature of leaflet tethering is different between inferior and anterior MI: asymmetric and predominant displacement of medial PM in inferior MI and symmetric displacement of both PMs in anterior MI.

\section{Relation to previous studies}

Multiple investigations have shown that basic mechanisms of ischemic MR is leaflet tethering by PM displacement with LV remodeling [8-21]. The present study confirmed the importance of leaflet tethering in the development of ischemic MR and further demonstrated different characteristics of leaflet tethering or asymmetric and symmetric PM displacements in patients with ischemic MR due to inferior and anterior MI.

Importance of asymmetric and symmetric PM displacements have been demonstrated in in vitro studies [26]. Symmetric PM tethering causes the coaptation line to shift apically but parallel to the annular plane, while asymmetric PM tethering causes an uneven coaptation line with greater leaflet displacement at the side of asymmetrically displaced PM. Kwan et al. recently demonstrated asymmetric and symmetric deformation of mitral leaflets in patients with inferoposterior MI and those with dilated cardiomyopathy, suggesting that the nature of leaflet tethering can be different according to the disease entity [27]. The pre- sent study confirmed these studies and further demonstrated heterogenous deformity of mitral valve complex or asymmetric and symmetric PM displacements in ischemic MR due to inferior and anterior MI.

\section{Clinical implications}

Different mechanisms of ischemic MR, presented in this study, highlight the importance of different or tailored strategies and procedures of treatment for individual patients. Although current annuloplasty is effective to repair ischemic MR [28, 29], the results can be suboptimal [30]. Interventions, which help restore the balance of bilateral tethering forces imposed on mitral leaflets, can be more efficient in patients with ischemic MR. Results of the current study encourages further development of procedures targeted to restore medial PM malposition in patients with inferior MI and those targeted to restore malpositions of both PMs in anterior MI.

\section{Limitations}

In this study, different natures of geometric distortion of the mitral valve apparatus in ischemic MR due to inferior and anterior MI were demonstrated. Characteristics of outward displacements of medial and lateral PMs were different between inferior and anterior MI. This study demonstrated utility of echocardiography to evaluate mitral valve pathophysiology [31]. However, antero-posterior, medio-lateral, and baso-apical components of PM displacements were not investigated. Further 3-dimensional study is necessary to clarify these spatial components $[32,33]$. Grade of ischemic MR was generally mild to moderate, and there was no patient with severe MR and regurgitant fraction $>50 \%$. This is due to the fact that grade of ischemic MR is usually mild in most patients [2]. Therefore, mechanisms of severe ischemic MR due to inferior and anterior MI were not investigated. However, it is still important to investigate the mechanism of mild to moderate ischemic MR because mild degree of ischemic MR is also a significant risk that affects patient prognosis [2]. Although the current study demonstrated different mechanisms of ischemic MR due to inferior and anterior MI, corresponding procedures to repair the mitral valve were not demonstrated. Multiple novel approaches to repair ischemic MR are under aggressive investigations [34-38]. However, approaches to correct deformed mitral valve apparatus, causing ischemic MR, are not established. Further investigations are required to establish efficient meth- 
ods to repair ischemic MR.

Nevertheless, understanding the pathophysiology is a prerequisite to successful repair of ischemic MR, and the purpose of this study was achieved by demonstrating different mechanisms of ischemic MR due to inferior and anterior MI, with potential applications for LV plasty targeted to heterogenous deformations of mitral valve apparatus in individual patients.

\section{References}

1. Czer LS, Gray RJ, DeRobertis MA, et al. Mitral valve replacement: impact of coronary artery disease and determinants of prognosis after revascularization. Circulation 1984; 70 (suppl I): I198-I207.

2. Grigioni F, Enriquez-Sarano M, Zehr KJ, Bailey KR, Tajik AJ. Ischemic mitral regurgitation: long-term outcome and prognostic implications with quantitative Doppler assessment. Circulation 2001; 103: 1759-64.

3. Lancellotti P, Troisfontaines P, Toussaint AC, Pierard LA. Prognostic importance of exercise-induced changes in mitral regurgitation in patients with chronic ischemic left ventricular dysfunction. Circulation 2003; 108: 1713-7.

4. Lamas GA, Mitchell GF, Flaker GC, et al. Clinical significance of mitral regurgitation after acute myocardial infarction. Circulation 1997; 96: 827-33.

5. Harris KM, Sundt TM III, Aeppli D, Sharma R, Barzilai B. Can late survival of patients with moderate ischemic mitral regurgitation be impacted by intervention on the valve? Ann Thorac Surg 2002; 74: 1468-75.

6. Trichon BH, Glower DD, Shaw LK, et al. Survival after coronary revascularization, with and without mitral valve surgery, in patients with ischemic mitral regurgitation. Circulation 2003; 108(Suppl II): II-103-II110.

7. Kisanuki A, Otsuji Y, Kuroiwa R, et al. Two-dimensional echocardiographic assessment of papillary muscle contractility in patients with prior myocardial infarction. J Am Coll Cardiol 1993; 21: 932-8.

8. Ogawa S, Hubbard FE, Mardelli TJ, Dreifus LS. Crosssectional echocardiographic spectrum of papillary muscle dysfunction. Am Heart J 1979; 97: 312-21.

9. Godley RW, Wann LS, Rogers EW, Feigenbaum H, Weyman AE. Incomplete mitral leaflet closure in patients with papillary muscle dysfunction. Circulation 1981; 63: 565-71.

10. Otsuji Y, Handschumacher MD, Schwammenthal E, et al. Insights from three-dimensional echocardiography into the mechanism of functional mitral regurgitation: direct in vivo demonstration of altered leaflet tethering geometry. Circulation 1997; 96: 1999-2008.

11. Komeda M, Glasson JR, Bolger AF, et al. Geometric determinants of ischemic mitral regurgitation.
Circulation 1997; 96 (Suppl II): II128-II133.

12. Otsuji Y, Handschumacher MD, Liel-Cohen N, et al. Mechanism of ischemic mitral regurgitation with segmental left ventricular dysfunction: three-dimensional echocardiographic studies in models of acute and chronic progressive regurgitation. J Am Coll Cardiol 2001; 37: 641-8.

13. Gorman RC, McCaughan JS, Ratcliffe MB, et al. Pathogenesis of acute ischemic mitral regurgitation in three dimensions. J Thorac Cardiovasc Surg 1995; 109: 684-93.

14. He S, Fontaine AA, Schwammenthal E, Yoganathan AP, Levine RA. Integrated mechanism for functional mitral regurgitation: leaflet restriction versus coapting force: in vitro studies. Circulation 1997; 96: 1826-34.

15. Nielsen SL, Nygaard H, Fontaine AA, et al. Chordal force distribution determines systolic mitral leaflet configuration and severity of functional mitral regurgitation. J Am Coll Cardiol 1999; 33: 843-53.

16. Messas E, Guerrero JL, Handschumacher MD, et al. Paradoxic decrease in ischemic mitral regurgitation with papillary muscle dysfunction: insights from three-dimensional and contrast echocardiography with strain rate measurement. Circulation 2001; 104: 1952-7.

17. Yiu SF, Enriquez-Sarano M, Tribouilloy C, Seward JB, Tajik AJ. Determinants of the degree of functional mitral regurgitation in patients with systolic left ventricular dysfunction: a quantitative clinical study. Circulation 2000; 102: $1400-6$.

18. Otsuji Y, Kumanohoso T, Yoshifuku S, et al. Isolated annular dilation does not usually cause important functional mitral regurgitation: comparison between patients with lone atrial fibrillation and those with idiopathic or ischemic cardiomyopathy. J Am Coll Cardiol 2002; 39: 1651-6.

19. Kumanohoso T, Otsuji Y, Yoshifuku S, et al. Mechanism of higher incidence of ischemic mitral regurgitation in patients with inferior myocardial infarction: quantitative analysis of left ventricular and mitral valve geometry in 103 patients with prior myocardial infarction. J Thorac Cardiovasc Surg 2003; 125: 135-43.

20. Gorman JH 3III, Gorman RC, Plappert T, et al. Infarct size and location determine development of mitral regurgitation in the sheep model. J Thorac Cardiovasc Surg 1998; 115: 615-22.

21. Timek TA, Lai DT, Tibayan F,et al. Ischemia in three left ventricular regions: insights into the pathogenesis of acute ischemic mitral regurgitation. J Thorac Cardiovasc Surg 2003; 125: 559-69.

22. Schiller NB, Shah PM, Crawford M, et al. Recommendations for quantification of the left ventricle by two-dimensional echocardiography. J Am Soc 
Echocardiogr 1989; 2: 358-67.

23. Vijayaraghavan G, Boltwood CM, Tei C, Wong M, Shah PM. Simplified echocardiographic measurement of the mitral annulus. Am Heart J 1986; 112: 985-91.

24. Ormiston JA, Shah PM, Tei C, Wong M. Size and motion of the mitral valve annulus in man. I. A two-dimensional echocardiographic method and findings in normal subjects. Circulation 1981; 64: 113-20.

25. Dujardin KS, Enriquez-Sarano M, Bailey KR, Nishimura RA, Seward JB, Tajik AJ. Grading of mitral regurgitation by quantitative Doppler echocardiography: calibration by left ventricular angiography in routine clinical practice. Circulation 1997; 96: 3409-15.

26. He S, Jimenez J, He Z, Yoganathan AP. Mitral leaflet geometry perturbations with papillary muscle displacement and annular dilatation: an in-vitro study of ischemic mitral regurgitation. J Heart Valve Dis 2003; 12: 300-7.

27. Kwan J, Shiota T, Agler DA, et al. Geometric differences of the mitral apparatus between ischemic and dilated cardiomyopathy with significant mitral regurgitation: realtime three-dimensional echocardiography study. Circulation 2003; 107: 1135-40.

28. Bach DS, Bolling SF. Improvement following correction of secondary mitral regurgitation in end-stage cardiomyopathy with mitral annuloplasty. Am J Cardiol 1996; 78: 966-9

29. Bolling SF, Pagani FD, Deeb GM, Bach DS. Intermediate-term outcome of mitral reconstruction in cardiomyopathy. J Thorac Cardiovasc Surg 1998; 115: 381-6.

30. Calafiore AM, Gallina S, Di Mauro M, et al. Mitral valve procedure in dilated cardiomyopathy: repair or replacement? Ann Thorac Surg 2001; 71: 1146-53.

31. Sonoda M, Takenaka K, Uno K, Ebihara A, Nagai R. The relation of mitral valve morphology to severe mitral regurgitation complicated with mitral valve prolapse. J Echocardiogr 2008; 6: 1-8.

32. Watanabe N, Ogasawara Y, Saito K, Yamaura Y, Tsukiji M, Okahashi N, Obase K, Okura H, Yoshida K. Quantitation of the degree of mitral valve prolapse by novel software system: new insights from transthoracic real-time three-dimensional echocardiography. J Echocardiogr 2007; 5: 112-7.

33. Okahashi N, Watanabe N, Yamaura Y, Wada N, Neishi Y, Kawamoto T, Toyota E, Okura H, Ogasawara Y, Yoshida $K$. Noninvasive measurements of the mitral annulus geometry by newly-developed quantitation software with real-time three-dimensional echocardiography: a validation study. J Echocardiogr 2007; 5: 32-7.

34. Liel-Cohen N, Guerrero JL, Otsuji Y, et al. Design of a new surgical approach for ventricular remodeling to relieve ischemic mitral regurgitation: insights from 3dimensional echocardiography. Circulation 2000; 101: 2756-63.

35. Messas E, Guerrero JL, Handschumacher MD, et al. Chordal cutting: a new therapeutic approach for ischemic mitral regurgitation. Circulation 2001; 104: 1958-63.

36. Hung J, Guerrero JL, Handschumacher MD, Supple G, Sullivan S, Levine RA.. Reverse ventricular remodeling reduces ischemic mitral regurgitation: echo-guided device application in the beating heart. Circulation 2002; 106: 2594-600.

37. Power JM, Raman J, Dornom A, et al. Passive ventricular constraint amends the course of heart failure: a study in an ovine model of dilated cardiomyopathy. Cardiovasc Res 1999; 44: 549-55.

38. Menicanti L, Di Donato M, Frigiola A, et al. Ischemic mitral regurgitation: intraventricular papillary muscle imbrication without mitral ring during left ventricular restoration. J Thorac Cardiovasc Surg 2002; 123: 1041-50. 